\title{
Nowhere to Go? Examining Facility Acceptance Levels for Serving Individuals Using Medications for Opioid Used Disorder
}

\author{
Nancy J. Kepple ${ }^{\mathrm{a}}$, Amittia Parker ${ }^{\mathrm{a}}$, Susan Whitmore ${ }^{\mathrm{b}}$, \& Michelle Comtois ${ }^{\mathrm{b}}$ \\ ${ }^{a}$ University of Kansas, School of Social Welfare, 1545 Lilac Lane, Lawrence, KS 66045, USA \\ ${ }^{b}$ First Call Alcohol/Drug Prevention \& Recovery, 9091 State Line Rd, Kansas City, MO 64114, \\ USA
}

Corresponding Author:

Nancy J. Kepple

KU School of Social Welfare

1545 Lilac Lane, 113 Twente Hall

Lawrence, KS 66045

USA

E-mail: njkepple@ku.edu

Telephone: (785) 864-0147 


\section{Introduction}

Opioid-related overdoses and deaths continue to increase across the United States, straining the substance use disorder (SUD) service sector with a growing need for recoveryoriented services and recovery support services (Hedegaard, Warner, \& Minino, 2017; Rudd, 2016). For the purpose of this study, we define recovery-oriented services as therapeutic services (including both medication and behavioral therapy) that occur after SUD diagnosis, ranging from Level 1 (Outpatient) to Level 4 (Medically Managed Inpatient) interventions (American Society of Addiction Medicine, 2015). Recovery support services are non-clinical, SUD-focused services that address dimensions of an individual's health, home, purpose, and community to help them maintain SUD treatment gains over time (Kaplan, 2008). Access to appropriate recoveryoriented and recovery support services may be key to reducing opioid-related harms with studies indicating significantly reduced risks for overdoses and deaths among individuals with opioid use disorders (OUD) who are receiving services rather than not receiving services (Sordo et al., 2017; Schwartz et al., 2013). For this population, access to medications for OUD is associated with better treatment outcomes and subsequently overall health outcomes (Volkow \& Wargo, 2018). Pharmacotherapies, including methadone, buprenorphine and naltrexone, help retain individuals in services and reduce risks for opioid-related harms, overdose, and death (Sordo, et al., 2017; Mattick, Breen, Kimber, \& Davoli, 2014; Nunes et al., 2018).

In light of these benefits, much of the extant literature has focused on understanding how to expand access to these medications (McElrath \& Joseph, 2018; Volkow \& Wargo, 2018). Recent policy and funding initiatives through Medicaid expansion and the Comprehensive Addiction and Recovery Act (2016) have supported the increased availability of medications for OUD through funding streams developed to address this growing epidemic (Andrilla, Moore, 
Patterson, \& Larson, 2018; Sharp et al., 2018). Yet, expanding access and use of these pharmacotherapies means little if individuals using them for their OUD are not concurrently able to access complementary recovery-oriented and recovery support services inherent to best practices for medication assisted treatment protocols (Kresina \& Lubran, 2011; National Institute on Drug Abuse, 2018; Robinson \& Adinoff, 2018). In fact, promoting access to on-going, comprehensive and holistic services is critical for this population given the highest risks for overdose occur both before and after treatment (Sordo et al., 2017). In other words, these individuals using medications for OUD would benefit from engaging in complementary recovery-oriented services and recovery support services to minimize their experiences of opioid-related harms. However, these services must exist and be willing to serve these individuals in order for them to benefit from receiving these services.

Existing studies provide initial insight into how the facilities providing SUD-related services may be responding to service-seeking individuals who are prescribed medications for OUD. Several facility-level factors may contribute to whether or not a facility decides to provide services to individuals using these medications, including services infrastructure, stigma, service philosophy, and client demographics (Chang, Klimas, Wood, \& Fairbairn, 2017; Hadland et al., 2018; Livingston, Adams, Jordan, MacMillan, \& Hering, 2017; Majer et al., 2018; McElrath, 2018; Roman, Abraham, \& Knudsen, 2011). For example, medical professionals on staff at facilities are often reluctant to prescribe and/or monitor medications for OUD due to their fears of working with opioid-using individuals, lack of knowledge about OUD, and/or concerns about surveillance duties, potentially limiting availability and support for medications for OUD at a given facility (Livingston et al., 2017; McElrath, 2018). It is less clear how these factors may affect provider preferences related to their openness to working with individuals using 
medications for OUD within the facilities providing less intensive recovery-oriented services and/or recovery support services. One study observed individuals within democratically-run, Oxford House settings (defined as a community-based approach which is participant-run and provides a supportive, sober living environment) conveyed predominantly mixed-to-negative attitudes towards use of these medications among residents (Majer et al., 2018). The presence of stigma against individuals using medications for their OUD suggest abstinence-based recovery homes may not be optimal resources of individuals using these medications (Majer et al., 2018). These findings also reflect the concerns proposed by Roman and colleagues (2011) about the lack of alignment between use of medications for OUD and both 12-step practices and traditional drug- and alcohol-free settings. Client demographics may also influence facility-level acceptance for medication use. For example, providers often underuse medication assisted treatment protocols with youth likely due to provider concerns about our limited understanding of the utility of medications for OUD with youth (Chang et al., 2017; Hadland et al., 2018).

This study aimed to gain further clarity on how facility-level characteristics may be contributing to service availability for individuals using medications for OUD and seeking complementary recovery-oriented services and/or recovery support services. First, we examined the level of a facility's acceptance of medications for OUD, defined by their willingness to provide services to individuals prescribed these medications and their level of involvement in managing the use of these medications. Second, we examined the facility-level characteristics associated with differences in facility acceptance of medications for OUD. Finally, we describe the reasons provided by facility representative about their rationale for a given medication acceptance levels. For this study, we did not capture finer grain detail about daily practices that may hinder treatment progress beyond service access. 


\section{Material and methods}

\subsection{Census of facilities providing clinical and non-clinical services to address substance use}

We used a university-agency collaboration model to conduct a needs assessment of a 10county region within the Kansas City metropolitan area. It arose out of response from the Kansas City Opioid Treatment Work Group identifying a need for regionally specific data about service capacity, particularly in relation to addressing OUD-related needs, in order to minimize harms of increasing rates of opioid use in the region. We aimed to capture a point-in-time assessment of the substance use disorder (SUD) service sector between Fall 2017 and Spring 2018. We defined the sector broadly to include all services that provided support to individuals at risk of substance misuse, actively misusing alcohol and/or other drugs, or in recovery from a SUD. We collected multiple listings for organizations that provided at least one of the following services: prevention/education services; screening/assessment services; providers of medications for OUD; detoxification services; intervention specialists; SUD treatment services (i.e., all levels of care from inpatient to traditional outpatient); recovery support services (defined by provision of sober housing, peer supports, recovery coaching and/or management, and community supports); and other non-SUD support services. In addition to understanding the context of services, we used this information to update the referral database for a SUD-related crisis hotline and an associated online resource guide hosted by our community partner.

As a first step, we obtained well-established referral listings in the metropolitan area that included United Way 211, My Resource Connection (Johnson County), Kansas Department of Aging and Disability Services (KDADS) Treatment Facility Summary, Missouri Department of Mental Health (DMH) Listings of Eligible Providers, and First Call Alcohol/Drug Prevention \& Recovery Resource Guide. In addition, we pulled listings from the following online directories: 
Substance Abuse and Mental Health Services Administration (SAMHSA) treatment locator, Oxford Houses, University of Kansas Medical Center (KUMC) Resource List, National Directory of Drug and Alcohol Abuse Treatment Listing, Addicted.org, DrugRehab.org, Recovery.org, FreeAddictionCenters.org, and Psychology Today. We obtained a listing of approved providers of medications for OUD from SAMHSA. To fill potential gaps within these directories, we also met with local representative of Kansas, Missouri, and Federal government agencies. In addition, we actively participated in three local task force/coalition meetings that included providers from multiple sectors serving substance-using populations. We also reached out to leaders within the field and leaders of agencies conducting innovative practices, who we identified through local media outlets, coalition meetings, and discussions with other key informants. Our goal was to obtain a representation of viewpoints throughout organizations providing services along the SUD continuum of care that ranged from screening and diagnosis to service linkages to recovery-oriented services to recovery support services. As a result, we spoke to representatives from the following groups: (1) government, private, non-profit, and faith-based organizations; (2) representatives from both Kansas and Missouri service sectors; and (3) providers offering a diverse range of services. We conducted 30 key informant interviews (10\% government representatives, $20 \%$ non-profit executive leadership, $10 \%$ faith-based executive administrators, $10 \%$ social services executive administrators, $10 \%$ prescribing physicians, $10 \%$ prevention specialists, $10 \%$ dual diagnosis providers, and $20 \%$ recovery support services providers).

As a second step, we de-duplicated the facilities identified from each of these sources to create a final list of 692 unique facility locations that were within the 10 county region. Finally, we followed procedures to obtain information from a maximum of five different source to verify 
that each location on our list was active and providing services to clients between September 2017 and March 2018. To track information consistently across sources, we created a long-form and short-form survey tool that aligned with the 2016 National Survey of Substance Abuse Treatment Services (NSSATS; SAMHSA, 2017). We also incorporated information from stakeholder interviews when relevant to understanding facility-level practices. In the end, we validated facility location and services through one or more of the following sources: (1) facility survey based on the 2016 version of the National Survey of Substance Abuse Treatment Services (NSSATS; SAMHSA, 2017) that was completed by facility directors; (2) web scrapes of agency websites with a follow-up telephone survey (using a modified short-form of the facility survey) with intake service specialists; (3) SAMSHA locator details updated in January 2017 using information from the 2016 NSSATS (SAMHSA, 2016); (5) stakeholder interviews; and/or (4) state summaries of eligible SUD services by location.

We identified 216 agencies comprising 410 facility locations that served individuals with substance-related needs across 10 counties that captured a mix of urban and rural geographies. Our verification process included confirmation that that the location was currently in operation by at least one source. Most responses were similar across data sources; however, if there was a difference in information across sources, we prioritized the information that reflected the most up-to-date information. For example, information collected from a facility representative between September 2017 to March 2018 through our survey methods would be prioritized over data collected from an agency website or the 2016 N-SSATS. In sum, 166 (40.4\%) were verified using 3 or more of the data sources; $230(56.1 \%)$ were verified using 2 of the data sources; and $14(3.4 \%)$ were verified using 1 of the data sources. This study excluded the $50(12.2 \%)$ facilities reporting that they do not treat opioid-related concerns and included the $360(87.8 \%)$ facilities 
reporting they provide services to clients with opioid-related concerns. Table 1 provides a description of the characteristics for all facilities that reported a willingness to treat clients with opioid-related concerns $(\mathrm{N}=360)$.

\section{$<$ INSERT TABLE 1 ABOUT HERE $>$}

\subsection{Measures}

Facilities reported on their ability and/or willingness to serve individuals using medications for OUD on a 4 point scale that we modified from the National Survey of Substance Abuse Treatment Service that ranges from 1 - Medications for OUD use not allowed and/or does not address opioid-related concerns to 4 - Medications for OUDs administered or monitored on site (N-SSATS; SAMHSA, 2016). In addition, we documented when facilities reported that they do not accept clients with opioid-related concerns due to a prevention-only focus or not serving clients with opioid-used disorders to help distinguish them from facilities serving clients with opioid-related concerns. Excluding facilities that do not address opioid-related concerns, these data were recoded as follows: 1 - zero acceptance (medications for OUD use not allowed); 2 low acceptance (medications for OUD use accepted with additional restrictions and/or reservations); 3 - moderate acceptance (medications for OUD use accepted without any restrictions or reservations); and 4 - high acceptance (medications for OUDs administered and/or monitored on site). For facilities reporting administering and/or monitoring these medications on site, we also collected the specific types of opioid-related medications (i.e., methadone, buprenorphine, or naltrexone); however, we did not track more specific information, such as mode of administration. Table 2 provides a summary of these distributions. Please note that we recoded the facility as having low acceptance if it only accepted clients on some types of medications for OUD (i.e., injectable vivitrol) but not other types (e.g., methadone). If a facility was willing to administer and monitor any type of medications for OUD, we recoded the facility 
as high acceptance given authorizations are required for providers to prescribed specific types of medications. In addition, we asked facilities to provide qualitative feedback explaining their selfreported acceptance level of medications for OUD via text in the online survey and verbally in the telephone-based survey (long and short form).

\section{$<$ INSERT TABLE 2 ABOUT HERE $>$}

Given the study's focus on assessing a broad range of facilities providing SUD-related services, we assessed for facility type and facility focus. Facilities reported the legal designation for their agency/organization for taxation purposes: for profit (1), nonprofit (2), faith based organization (3), or government-run (4). Each facility also reported on the primary treatment focus of their location: substance use disorder (SUD) treatment (1), mental health or mixed mental health/SUD treatment (2), general health (3), or other social services (4).

The Midwestern region where we conducted the study has a large presence of faithoriented service providers within the SUD treatment field. To capture potential differences by spiritual orientation of actual practices, we asked facilities to rate the level of spiritual orientation that aligns with their daily services using the typology presented by Sider \& Unruh (2004). Each facility reported on the spiritual characteristics of their facility by rating it as 1 - secular (agency has no religious identity or content); faith-partnership (agency has a partnership with a faithoriented organization or funder); faith-background (agency have historical ties to a faith or faithoriented organization but operates in more of a secular model); faith-affiliated (agency may have little to no religious content but does encourage religious participation); faith-centered (agency is explicitly religious, but participants can opt-out of religious activities); or faith-permeated (agency requires participation in religious elements). Based on the distribution of categories, we operationalized spiritual orientation as a categorical variable to distinguish between purely 
secular (1) facilities from those with some faith orientation ranging from faith-affiliated to faith permeated (0).

We documented availability of non-English language services, eligible ages for services, and eligible gender for services for each facility. We operationalized language services as a dichotomous variable for English Only (1) and English plus other language (0). We dichotomized age-specific services into adult only (1) and inclusion of other age groups, such as adolescents (0). We operationalized gender-specific services as a categorical variable using three categories: women only (1), men only (2), and both women and men (3).

Facilities often provided multiple SUD services; as a result, we created non-mutually exclusive dichotomous variables for SUD services by level of care, guided by the American Society for Addiction Medicine's level of care system (ASAM, 2015). Services included medical detox, acute inpatient, residential treatment, day treatment/partial hospitalization, intensive outpatient, traditional outpatient, and recovery support services. Based on the study criteria, screening/prevention/education services were excluded from this study due to the inability to address opioid-specific concerns. Due to a low number of facilities reporting medical detox or acute inpatient services, these services were combined into one category that represented services provided within hospital settings, resulting in a possible range of 1 to 6 SUD services per facility. We also created a count for the total number of non-SUD services. Non-SUD services were categorized into ten possible services that reflect NIDA-recommended care that help to facilitate holistic and individualized treatment plans (NIDA, 2018): health/education services, mental health/counseling services, employment/education support, legal services, case management for social services referral or instrumental supports, domestic violence services, housing services that provided or located shelters or permanent housing, transportation services, 
parenting supports such as child care or parenting education, and family psychoeducation and supports. We created a count for total number of non-SUD services per facility, with a possible range from 0 to 10 .

Given the chosen Metropolitan area is bisected by a state line, we controlled for the State (coded at State 1 and State 2) where the facility is located given substantial differences in policies related to funding and initiatives promoting MAT prescription and distribution.

\subsection{Analytic Procedures}

For the quantitative data, we ran descriptive statistics and chi-square tests for independence. We used Stata/SE 14.0 to conduct unadjusted and adjusted multinomial logistic regression for our multivariate analyses; we chose this model to avoid potential bias from assuming parallel regression across categories as observed with ordinal logistic regression models (Long \& Freese, 2006). We used a mlogit command in Stata 14/SE to simultaneously estimate binary logits for acceptance categories (i.e., zero, low, and high) compared to the base outcome of moderate acceptance (Long \& Freese, 2006). We evaluated goodness of fit and conducted regression diagnostics using Stata/SE 14.0 (Long \& Freese, 2006); and we ran the mlogitroc module to assess for model accuracy (Peterson, 2010). Coefficients from the model were exponentiated $\left(\mathrm{e}^{\mathrm{coef}}\right)$ to obtain relative risk ratios (rrr). Our final analytic sample was composed of 350 facilities with complete information. The $10(2.8 \%)$ cases with missing data were deleted case-wise. All data appeared to be missing at random with no significant differences observed between cases with and without missing data.

The final multivariate model included facility focus, adult only population, recovery support services, multiple SUD services, multiple non-SUD services, and a control for state where the facility is located. To minimize multicollinearity, gender-specific service was removed 
from the model as this variable was associated with recovery support services (primarily driven by housing services) and was destabilizing the model. In addition, agency type was removed due to collinearity with facility focus; facility focus was maintained given it gave a more precise description of facility-specific activity. We removed the variables measuring language services, presence of SUD services less than 20 hours/week, and presence of SUD service more than 20 hours/week from the final model due to collinearity with the variable measuring facilities with multiple SUD services. We retained the variable for multi-SUD services given it better portrays global SUD service capacity than any one of the other individual variables. In the unadjusted models, the distribution of faith-orientation resulted in inflated standard errors due to a small proportion of agencies reporting non-secular orientation within high acceptance facilities. Due to this issue in addition to $79.5 \%$ of all faith-oriented facilities reported providing recovery support services $(\chi 2(1)=34.30, p<.001)$, we decided to remove this variable from the final model to improve model fit.

For the qualitative data, we conducted a content analysis with a final analytic sample of 89 facilities providing rationale for their choice (Neuendorf, 2017). We used content analysis to help summarize a large number of brief, qualitative responses and to identify trends in rationales for decisions across the acceptance categories (Bengtsson, 2016; Neuendorf, 2017). After extracting the qualitative responses, two members of the research team coded the data using Excel and Nvivo 12. We then used multiple rounds of close reading of the text to inductively identify categories and developed an associated codebook with definitions of what rationales fell into each category. Specifically, the first round of coding focused on descriptive, in vivo, and heuristic coding, such as "insufficient supports" (Miles, Huberman, \& Saldana, 2014). Subsequent second round coding developed pattern codes including implicit themes revolving 
around facility acceptance. We were unable to obtain qualitative feedback from $75.3 \%$ of facilities, which represents a substantial amount of missing data. Data was not missing at random with significant differences observed between facilities providing and not providing their rationale for the self-reported acceptance level of medications for OUD. Specifically, facilities providing rationale were observed to be composed of a higher proportion of low acceptance (44.9\% compared to $6.1 \% ; \mathrm{x} 2(3)=78.83, p<.001)$. It may be that representative reporting low acceptance felt compelled to justify the complex nature of their approach to serving clients using medications for OUD. In addition, facilities providing qualitative feedback were observed to have a lower proportion of secular orientation $(53.9 \%$ compared to $82.0 \%$; $\mathrm{x} 2(1)=27.76, \mathrm{p}<$ $.001)$; a higher proportion providing recovery support services (66.3\% compared to $47.9 \%$; $\times 2(1)$ $=9.01, \mathrm{p}=.003)$; or a lower proportion providing adult only services $(49.4 \%$ compared to $68.2 \% ; \mathrm{x} 2(1)=10.07, p=.002)$. Given the low response rate to this question, we view these data as exploratory with limited generalizability, and we primarily used these data to contextualize the quantitative findings.

\section{Results}

\subsection{Bivariate Analyses}

Table 1 and 2 show the univariate distributions of facility characteristics for all 360 facilities identifying as serving clients with opioid-related needs. Among these, we observed high acceptance facilities reported some variation in the types of medications they were authorized to prescribe. Of the 90 facilities reporting ability and willingness to administer and/or monitor medications for OUD, 8.9\% $(\mathrm{n}=8)$ identified as Opioid Treatment Programs administering methadone. Most facilities administering methadone also administered either buprenorphine $(\mathrm{n}=$ 4) or naltrexone $(\mathrm{n}=2)$. In addition, $53.3 \%(\mathrm{n}=48)$ reported prescribing buprenorphine only, 
$17.7 \%(n=16)$ reported prescribing naltrexone only, and 20.0\% $(n=18)$ reported prescribing both buprenorphine and naltrexone.

Table 3 shows the bivariate comparisons of several facility characteristics with acceptance level for the 350 facilities with complete data. First, approximately $25 \%(\mathrm{n}=88)$ of the 350 facilities used in the analytic sample that were open to serving clients with opioid-related concerns reported zero acceptance for clients using medications for OUD. However, the proportion of zero acceptance facilities varied by significantly by level of care with $0 \%$ of facilities identifying as zero acceptance in facilities offering inpatient or medical detox services compared to over $40 \%$ for facilities offering recovery support services.

\section{$<$ INSERT TABLE 3 ABOUT HERE $>$}

In addition, we observed a higher proportion of high acceptance facilities identified as being a part of private, for-profit organizations, likely driven by a higher proportion of these providers identifying as private medical providers. We observed a higher proportion of facilities that focused primarily on mental health and general health also identified as moderate and high acceptance facilities. In contrast, a higher proportion of agencies focused on SUD only or other services reported identifying as zero to low acceptance facilities. Acceptance level also appeared to significantly differ by type of services offered at a facility, including the number of SUD services and non-SUD services offered at a given facility location.

\subsection{Multivariate Analyses}

Table 4 shows the final adjusted model that demonstrated the best fit and alignment with model assumptions. The model results in three separate regressions that compare three acceptance level categories (i.e., zero acceptance, low acceptance, and high acceptance) to the base outcome of moderate acceptance. For example, the column labeled low acceptance 
represents the model estimating the probability that a facility reported low acceptance of individuals using medications for OUD $\left(\mathrm{P}_{\text {low }}\right)$ relative to the probability that a facility reported moderate acceptance of individuals using medications for OUD ( $\left.\mathrm{P}_{\mathrm{mod}}\right)$, or the relative risk of $\left(\mathrm{P}_{\text {low }}\right) /\left(\mathrm{P}_{\mathrm{mod}}\right)$. We report the relative risk ratios (rrr) in Table 4 , and one should interpret these as the ratio of relative risk for each facility characteristics similar to how we interpret odds ratios (Long \& Freese, 2006). The final model significantly differs from the intercept-only model with good model fit and model accuracy with an excellent fitting ROC $(\mathrm{AUC}=0.99)$. The final model also met the assumption for independence of irrelevant alternatives.

$<$ INSERT TABLE 4 ABOUT HERE $>$

We observed significant differences between zero and moderate acceptance facilities by facility characteristics. For facilities with a primary focus on mental health services relative to other types of primary focus (i.e., SUD only, general health, social services), the relative risk for identifying as zero acceptance (compared moderate acceptance) would be expected to decrease by a factor of 0.39 when all other variables in the model are held constant. In other words, facilities with a primary focus on mental health are less likely than facilities with another primary focus to identify as zero acceptance than moderate acceptance. All else being equal, the probability of a facility identifying as zero acceptance (compared to moderate acceptance) is higher for facilities providing recovery support services (compared to those not providing these services); however, identification as a zero acceptance facility was lower for facilities that provided multiple SUD services (compared to only one SUD service). We also observed the facilities in one state (that did not have policies promoting acceptance of individuals using medications for OUD) were more likely than the other state (that had recently implemented funding and policies promoting acceptance of individuals using medications for OUD) to identify as zero acceptance than moderate acceptance. 
We observed two important distinctions between low acceptance facilities that accepted individuals using medications for OUD with reservations and/or additional restrictions and moderate acceptance facilities that accepted these individuals without reservations and/or additional restrictions. All else being equal, facilities offering recovery support services were more likely than those facilities not providing these services to identify as low acceptance than moderate acceptance. We also observed the relative risk for facilities identifying as a low acceptance facility compared to a moderate acceptance facility would be expected to decrease by 90\% for facilities providing multiple SUD services, holding all other variables constant.

Finally, the model compared high acceptance facilities to moderate acceptance facilities. The relative risk of a facility administering and/or monitoring medications OUDs on site compared to just being unreservedly open to serving individuals using these medications increases by a factor of 3.22 for facilities that identifying as having a primary mental health focus. For facilities serving adults only relative to being open to serving children and adolescents, the relative risk for being a high acceptance facility (compared to moderate acceptance) would be expected to increase by $412 \%$ given the other variables in the model are held constant. Opposite to what we observed for the model comparing low acceptance to moderate acceptance facilities, we observed that facilities offering recovery support services were less likely than those facilities not providing these services to identify as high acceptance than moderate acceptance. In addition, facilities with multiple SUD services were more likely than facilities with only one SUD service to identify as high acceptance compared to moderate acceptance.

\subsection{Content Analysis}


Table 5 provides a summary of categories and subcategories that we identified from the 89 qualitative responses that we received from facility representatives justifying their facility's acceptance level. We used this information to help us gain insight into additional factors, beyond facility level characteristics measured by our survey tools, which may contribute to the observed variation in facility acceptance of medications for OUD.

$<$ INSERT TABLE 5 ABOUT HERE $>$

\subsubsection{Alcohol/drug free facility. Facilities reporting use of an alcohol/drug free} environment fell into two groups: 1) explicit statements that no narcotics were allowed and that they viewed medications for OUD as narcotics, and 2) more general concerns about potential negative impact on the therapeutic community. When looking at rationale by facility characteristics, we observed alcohol- and drug-free facilities where "no narcotics" were allowed all identified as faith-oriented facilities $\left(\chi^{2}(1) 13.19, p<.001\right)$ and as not accepting clients using medications for OUD $\left(\chi^{2}(3)=51.40, p<.001\right)$. Many described that they "follow a traditional 12-step model; no narcotics" or "do not allow any narcotics use; zero tolerance". In contrast, facilities that low acceptance of individuals using medications for OUD reported additional monitoring and/or stricter rules were in place to ensure the therapeutic community was not adversely affected by the behavior of an individual using these medications. For example, facilities described beliefs similar to having "reservations about how medications may impact the community, given their psychotropic effects. Strict rules applied to this group."

3.4.2 Provider preference. A high proportion of facilities reporting provider preference also reported a mental health focus $\left(69.2 \% ; \chi^{2}(2)=7.41, p=.025\right)$, being secular in orientation $\left(92.3 \% ; \chi^{2}(1)=9.02, p=.003\right)$, and not providing recovery support services $\left(92.3 \% ; \chi^{2}(1)=\right.$ 23.40, $p<.001)$. We observed this explanation to be split between facilities reporting high 
acceptance (where providers actively chose to administer and/or monitor medications OUD) and facilities reporting reservations in acceptance that depended on individual provider comfort.

3.4.3 Drug Specific Preferences. Drug-specific preferences were predominantly reported by facilities providing recovery services $\left(\chi^{2}(1)=3.86, p=.049\right)$ and those reporting low acceptance of medications for OUD $\left(\chi^{2}(3)=9.31, p=.025\right)$. Facilities reported more subtle restrictions such as drug-specific preferences, varying from preference for "Methadone or Vivitrol" to more specific responses that "Vivitrol okay. Methadone or suboxone are problematic; there is no way to monitor them, and it opens up problems."

3.4.4 Prior Experiences. Prior experiences (whether good or bad) were associated with agency focus, specifically a lower proportion identifying as mental health focused (4.5\%) compared to other focus $\left(95.5 \% ; \chi^{2}(1)=12.52, p<.001\right)$ or as a multiservice facility $(9.1 \%)$ compared to single SUD service facility $\left(90.9 \% ; \chi^{2}(1)=9.16, p<.002\right)$. A few zero acceptance facilities specifically described not accepting individuals using medications for OUD "based on prior experience" with concerns. Low acceptance facilities that accepted individuals using medications for OUD with reservations and/or additional restrictions $(\mathrm{n}=50)$ primarily reported having bad prior experiences with individuals using medications for OUD: "It depends on the situation. We've had bad experiences with methadone and suboxone. It's also hard to tell use levels with a UA." On the flipside, several moderate acceptance facilities reported having good experiences with individuals using medications for OUD: "Women that are on medications do well here. They seem to really want to make a change for the better."

3.4.5 Level of Supports. Presence of sufficient supports aligns with moderate to high acceptance facilities. Sufficient supports were associated with agency focus on mental health $\left(\chi^{2}\right.$ $(1)=5.97, p=.015)$ with a higher proportion of facilities reporting sufficient supports also 
identifying as having a primary mental health focus $(62.5 \%)$ compared to other focus $(30.1 \%)$. Similar patterns were observed for facilities with secular orientations $\left(\chi^{2}(1)=12.45, p<.001\right)$ and provision of multiple SUD services $\left(\chi^{2}(1)=17.38, p<.001\right)$. Specifically, moderate acceptance facilities reported having psychiatrists on an advisory board, physicians that actively partner with the agency as an outside support, or specific therapeutic programming that supported use of medication for OUD like the Matrix Model or harm reduction philosophies. Along these lines, high acceptance facilities $(\mathrm{n}=8)$ described having sufficient supports for individuals using MATs, such as "full-time psychiatrists and medical doctors on site" were open to providing these services. These facilities reporting their rationale were predominantly multiservice facilities with some type of mental health focus with psychiatrists on staff, and they do not reflect the physicians providing standalone services.

In contrast, zero to low acceptance facilities specified that they has insufficient supports and/or training to assist individuals with needs that arise around medication management. This often resulted in these individuals being "referred out if medication needs present" in zero acceptance facilities. Many low acceptance facilities describe having "mixed reports about the effects of medications and having a lack of understanding regarding what medication assisted treatment involves" and many conveyed desire to only accept clients if their medication protocol and recovery was "well maintained".

\subsubsection{No Screening/Assessment for Medications for OUD. Several facilities reported} moderate acceptance due to the simple fact that they did not actively assess if clients were using of medications for OUD. These facilities all reported offering faith-oriented services $\left(\chi^{2}(1)=\right.$ $8.90, p=.003$ ) and reported a primary focus on social services, typically providing recovery support services in lieu of recovery-oriented services. 


\section{Discussion}

\subsection{Discussion of Findings}

Our study looked beyond availability of providers to prescribe medications for OUD to look at facility acceptance of these medications for any facility providing recovery-oriented or recovery support services open to clients with opioid-related needs. We identified a quarter of these facilities were unwilling to serve individuals using medications for OUD, particularly those focused on recovery support services. We also observed an additional $16 \%$ of low acceptance facilities (primarily less intensive recovery-oriented services like outpatient settings or recovery support services) who were willing to serve these individuals but may be providing less hospitable environments due to increased oversight and restrictions that these individuals are subject to due to their medication use. These observations build upon prior work that observed finding mixed-to-negative attitudes towards medication for OUD among residents of abstinencebased recovery homes (Majer et al., 2018; Roman et al., 2011) by highlighting that over 40\% of the SUD service sector in this Midwestern region were not fully open to accepting clients due to their medication use with mixed-to-negative attitudes. That being said, it is important to emphasize that factors contributing to these trends are complex and varied, providing multiple points for intervention at an organizational level to increase services for individuals using medications for OUD and in need of complementary recovery-oriented and recovery support services.

Multi-service facilities and/or facilities primarily focused on mental health or mixed mental health-SUD are more likely to report high acceptance or moderate acceptance of individuals using medications for OUD. These results align with qualitative feedback that these agencies report having sufficient infrastructure to support individuals on these medications (such 
as full-time psychiatrists on staff), even when receiving less intensive services. In contrast, it may be that smaller facilities focused on one type of SUD service that are less intensive than medical detox/inpatient settings are the most difficult to access for individuals using medications for OUD due to zero to low acceptance. This is particularly true for facilities offering recovery support services, such as sober living and peer navigation services, within this study's region. This finding is concerning given lower levels of care and recovery support services are critical during an individual's post-treatment stabilization in the community, and they can serve to reinforce medication compliance necessary for relapse prevention over time (Ma et al., 2018; Nunes et al., 2018).

Smaller, stand-alone facilities with fewer resources reported a lower likelihood of providing services to individual using medications due to concerns that they lacked the necessary tools to serve these individuals adequately. In addition, underlying stigma towards medications for OUD discussed in prior works persisted within our study's service sector (McElrath, 2018), particularly in facilities providing recovery support services, where representatives reported bad experiences of individuals using medications over the past 10 to 20 years. Facility settings based on traditional 12-step or moralistic models of SUD reinforced the stigma, with medications viewed as continued use of psychoactive substances. In fact, our qualitative feedback aligns with prior work that suggests provider preference not to treat individuals using medications for OUD also limits availability of services for these individuals (Livingston et al., 2017); to address the limited number of providers available to prescribe, monitor, and provide behavioral therapy goes beyond basic training needs and will require addressing provider perceptions and fears. The development of injectable naltrexone may have shifted some concerns regarding daily management of medication use. However, the preference for injectable naltrexone is somewhat 
concerning given the limited evidence base for this medication being more effective than methadone or buprenorphine (Lee et al., 2018; Tanum et al., 2017) and contradicts best practices to match treatment protocols to the individual needs of the client (NIDA, 2018).

\subsection{Strengths \& Limitations}

These findings should be interpreted with key strengths and limitations in mind. This study is limited to an assessment of one Midwestern metropolitan area, providing insight into service trends that may not be duplicated in other metropolitan areas in the United States but can serve as a starting point for exploration of critical gaps in serving the needs of individuals using medications for their opioid use disorder. In addition, this study identified and contacted a census of facilities in the metropolitan area providing services to populations at-risk for, in need of treatment for, or in recovery from SUDs. It is likely we did not capture facilities that were not listed in existing referral databases, did not participate in local coalitions, or were not licensed through state organizations. Specifically, the use of one question regarding acceptance of medications for OUD limits our understanding of how facilities representatives interpreted this question. To address this limitation, we asked a follow-up question to provide an opportunity for facilities to provide a justification for their acceptance level. However, missingness observed in the qualitative responses limits our generalization of these qualitative findings. Instead, we view the facilities' qualitative justifications as helping to provide some clarification regarding the complex range of concerns that may be driving these differences across facilities. The study also offset potential bias in facilities positively self-reporting their facility services by triangulating multiple sources of information, including engaging both facility-level directors in a formal survey and intake workers through a short-form survey. Finally, we did not survey or interview 
the consumers of these services. This data best represents the experiences and perspectives of policy-makers, funders, coalition members, and/or providers.

\subsection{Implications for Policy, Practice, \& Research}

To ensure minimizations of opioid-related harms, states must move beyond funding increased access to medications for OUD by also leveraging funding to build infrastructure to support the treatment and recovery needs of individuals with opioid use disorder across recovery-oriented care and recovery support services. For example, motivating psychiatrists and recovery services to collaborate through funding mechanisms that establish create partnerships across providers may initially address infrastructure concerns for smaller, stand-alone providers. Our findings suggest these partnerships may be particularly critical for recovery support services. These types of strategies can help to promote medication adherence that is associated with lowered relapse and mortality rates (Ma et al., 2018; Nunes et al., 20108). In addition, trainings need to target providers beyond the medical/mental health fields to address hesitations/concerns about medications of OUD. This form of outreach may be more effective through providing education about the benefits and harms associated with each medication, explicitly facilitating discussions about navigating the challenges that providers face when serving individuals using medications and exploring the tensions between abstinence only and harm reduction treatment models (Sederer \& Marino, 2018).

Future research would benefit from further exploring the factors identified with zero and low acceptance facilities that have emerged. For example, more information is needed to understand the history of self-reported "bad experiences" with medications for OUD. More indepth exploration may help policy makers, administrators, and providers better understand the sources of reservations or fears. In addition, further examination of this information can provide 
insight into the specific infrastructural and training needs among zero and low acceptance facilities.

\subsection{Conclusions}

To support the individuals who are increasingly being prescribed medications for OUDs, providers of recovery-oriented and recovery support services need to be actively engaged to identify potential hesitations or concerns about treating this population and to be educated about the medications available. Specifically, there is a need to develop funding for education, training, and infrastructure that actively address the gap in available services open to individuals using medications for OUD, particularly for facilities offering recovery support services. These services are critical to support individuals in their long-term recovery and reduce post-treatment relapse, overdose, and deaths. Without these changes, our efforts to expand access and use of medications for OUD may fall short as individuals being prescribed these medications have even fewer options of where they can obtain complementary therapeutic services and/or recovery support services.

Declarations of interest: None.

\section{Funding}

First Call Alcohol/Drug Prevention \& Recovery, First Call Needs Assessment: Assessing Capacity for Substance Use Disorder Treatment Capacity in the Greater Kansas City Area; Jackson County, Missouri Outside Agency Funding, First Call Technology Capacity and Opioid Treatment Resource Analysis; and University of Kansas, School of Social Welfare, PI Start-up Funds. 


\section{References}

American Society of Addiction Medicine (ASAM). (2015). What is the ASAM Criteria? [webpage]. Retrieved on January 1, 2018 from https://www.asam.org/resources/theasam-criteria/about

Andrilla, C. H. A., Moore, T. E., Patterson, D. G., \& Larson, E. H. (2019). Geographic Distribution of Providers With a DEA Waiver to Prescribe Buprenorphine for the Treatment of Opioid Use Disorder: A 5-Year Update. The Journal of Rural Health, 35(1), 108-112. doi: $\underline{10.1111 / \mathrm{jrh} .12307}$

Bengtsson, M. (2016). How to plan and perform a qualitative study using content analysis. NursingPlus Open, 2, 8-14. doi: 10.1016/j.npls.2016.01.001

Chang, D. C., Klimas, J., Wood, E., \& Fairbairn, N. (2018). A case of opioid overdose and subsequent death after medically supervised withdrawal: The problematic role of rapid tapers for opioid use disorder. Journal of Addiction Medicine, 12(1), 80-83. doi: $\underline{10.1080 / 00952990.2017 .1399403}$

Hadland, S. E., Bagley, S. M., Rodean, J., Silverstein, M., Levy, S., Larochelle, M. R., ... \& Zima, B. T. (2018). Receipt of timely addiction treatment and association of early medication treatment with retention in care among youths with opioid use disorder. JAMA Pediatrics, 172(11), 1029-1037. doi:10.1001/jamapediatrics.2018.2143

Hedegaard, H., Warner, M., \& Miniño, A. M. (2017). Drug overdose deaths in the United States, 1999-2016. NCHS data brief, no 273. Hyattsville, MD: National Center for Health Statistics. 
Kaplan, L., The Role of Recovery Support Services in Recovery-Oriented Systems of Care. DHHS Publication No. (SMA) 08-4315. Rockville, MD: Center for Substance Abuse Treatment, Substance Abuse and Mental Health Services Administration, 2008.

Kresina, T. F., \& Lubran, R. (2011). Improving public health through access to and utilization of medication assisted treatment. International Journal of Environmental Research and Public Health, 8(10), 4102-4117. doi:10.3390/ijerph8104102

Lee, J. D., Nunes Jr, E. V., Novo, P., Bachrach, K., Bailey, G. L., Bhatt, S., ... \& King, J. (2018). Comparative effectiveness of extended-release naltrexone versus buprenorphinenaloxone for opioid relapse prevention (X: BOT): a multicentre, open-label, randomised controlled trial. The Lancet, 391(10118), 309-318.

Livingston, J. D., Adams, E., Jordan, M., MacMillan, Z., \& Hering, R. (2018). Primary care physicians' views about prescribing methadone to treat opioid use disorder. Substance Use \& Misuse, 53(2), 344-353. doi:10.1080/10826084.2017.1325376

Long, J.S. (1997). Regression Models for Categorical and Limited Dependent Variables. Thousand Oaks, CA: Sage Publications, Inc.

Long, J. S., \& Freese, J. (2006). Regression Models for Categorical Dependent Variables Using Stata ( $\left.2^{\text {nd }} E d.\right)$. College Station, Tx: Stata Press.

Majer, J. M., Beasley, C., Stecker, E., Bobak, T. J., Norris, J., Nguyen, H. M., ... \& Jason, L. A. (2018). Oxford House residents' attitudes toward medication assisted treatment use in fellow residents. Community Mental Health Journal, 54, 571-578. doi:10.1007/s10597017-0218-4 
Mattick, R. P., Breen, C., Kimber, J., \& Davoli, M. (2014). Buprenorphine maintenance versus placebo or methadone maintenance for opioid dependence. Cochrane Database of Systematic Reviews. doi:10.1002/14651858.CD002207.pub4

McElrath, K. (2018). Medication-Assisted Treatment for Opioid Addiction in the United States: Critique and Commentary. Substance Use \& Misuse, 53(2), 334-343. doi: $10.1080 / 10826084.2017 .1342662$

McElrath, K., \& Joseph, H. (2018). Medication-assisted treatment (MAT) for opioid addiction: Introduction to the special issue. Substance Use \& Misuse, 52(2), 177-180. doi: $10.1080 / 10826084.2017 .1404106$

Miles, M. B., Huberman, A. M., \& Saldana, J. (2014). Qualitative Data Analysis. Thousand Oaks, CA: Sage Publications, Inc.

National Institute on Drug Abuse. (2018). Principles of Drug Addiction Treatment: A Researchbased Guide ( $3^{\text {rd }}$ Ed.). Bethesda, MD; National Institute on Health, U.S. Department of Health and Human Services.

Neuendorf, K. A. (2016). The Content Analysis Guidebook. Thousand Oaks, CA: Sage Publications, Inc.

Robinson, S. M., \& Adinoff, B. (2018). The mixed message behind "Medication-Assisted Treatment" for substance use disorder. The American journal of drug and alcohol abuse, 44(2), 147-150. doi: 10.1080/00952990.2017.1362419

Roman, P. M., Abraham, A. J., \& Knudsen, H. K. (2011). Using medication-assisted treatment for substance use disorders: Evidence of barriers and facilitators of implementation. Addictive Behaviors, 36(6), 584-589. doi:10.1016/j.addbeh.2011.01.032 
Rudd, R. A. (2016). Increases in drug and opioid-involved overdose deaths-United States, 2010-2015. MMWR. Morbidity and mortality weekly report, 65, 1445-1452. doi:10.15585/mmwr.mm655051e1

Schwartz, R. P., Gryczynski, J., O’Grady, K. E., Sharfstein, J. M., Warren, G., Olsen, Y., ... \& Jaffe, J. H. (2013). Opioid agonist treatments and heroin overdose deaths in Baltimore, Maryland, 1995-2009. American Journal of Public Health, 103(5), 917-922.

Sharp, A., Jones, A., Sherwood, J., Kutsa, O., Honermann, B., \& Millett, G. (2018). Impact of Medicaid Expansion on Access to Opioid Analgesic Medications and MedicationAssisted Treatment. American Journal of Public Health, 108(5), 642-648.

Sider, R. J., \& Unruh, H. R. (2004). Typology of religious characteristics of social service and educational organizations and programs. Nonprofit and Voluntary Sector Quarterly, 33(1), 109-134. doi:10.1177/0899764003257494

Sordo, L., Barrio, G., Bravo, M. J., Indave, B. I., Degenhardt, L., Wiessing, L., ... \& PastorBarriuso, R. (2017). Mortality risk during and after opioid substitution treatment: systematic review and meta-analysis of cohort studies. $B M J, 357, \mathrm{j} 1550$. doi:10.1136/bmj.j1550

Substance Abuse and Mental Health Services Administration (2017). National Survey of Substance Abuse Treatment Services (N-SSATS): 2016. Data on Substance Abuse Treatment Facilities. BHSIS Series S-93, HHS Publication No. (SMA) 17-5039. Rockville, MD: Substance Abuse and Mental Health Services Administration.

Tanum, L., Solli, K. K., Benth, J. Š., Opheim, A., Sharma-Haase, K., Krajci, P., \& Kunøe, N. (2017). Effectiveness of injectable extended-release naltrexone vs daily buprenorphine- 
naloxone for opioid dependence: a randomized clinical noninferiority trial. JAMA psychiatry, 74(12), 1197-1205.

Volkow, N. D., \& Wargo, E. M. (2018). Overdose Prevention Through Medical Treatment of Opioid Use Disorders. Annals of Internal Medicine. 169(3),190-192. doi:10.7326/M181397. 
RUNNING HEAD: MOUD Acceptance

Table 1: Characteristics of Facilities Willing to Serve Clients with Opioid-related Needs $(N=360)$

\begin{tabular}{|c|c|}
\hline Facility Characteristics & Count (\%) \\
\hline \multicolumn{2}{|l|}{ Facility type (legally defined for taxation purposes) } \\
\hline Private, for profit & $134(37.2)$ \\
\hline Private, nonprofit & $198(55.0)$ \\
\hline Other & $28(7.8)$ \\
\hline \multicolumn{2}{|l|}{ Facility focus } \\
\hline SUD prevention/treatment only & $170(47.2)$ \\
\hline $\mathrm{MH}$ treatment or mixed $\mathrm{MH} / \mathrm{SUD}$ treatment & $142(39.4)$ \\
\hline General health & $9(2.5)$ \\
\hline Other & $39(10.8)$ \\
\hline \multicolumn{2}{|l|}{ Level of spiritual orientation (practice-defined) } \\
\hline Secular & $266(74.3)$ \\
\hline Faith partnership, background, or affiliation & $10(2.8)$ \\
\hline Faith background & $5(1.4)$ \\
\hline Faith affiliation & $19(5.3)$ \\
\hline Faith-centered & $37(10.3)$ \\
\hline Faith-permeated & $21(5.9)$ \\
\hline Missing & $2(0.0)$ \\
\hline \multicolumn{2}{|l|}{ Language services } \\
\hline English only & $107(29.7)$ \\
\hline English plus other language services & $253(70.3)$ \\
\hline \multicolumn{2}{|l|}{ Age-specific services } \\
\hline Adult only (ages 18+ years) & $133(36.9)$ \\
\hline Other ages (adolescent also served) & $227(63.1)$ \\
\hline \multicolumn{2}{|l|}{ Gender-specific services } \\
\hline Women only & $42(11.7)$ \\
\hline Men only & $67(18.7)$ \\
\hline Both women \& men & $250(69.6)$ \\
\hline Missing & $1(0.0)$ \\
\hline \multicolumn{2}{|l|}{ Accept Medicaid/Medicare reimbursement? } \\
\hline No & $158(43.9)$ \\
\hline Yes & $116(32.2)$ \\
\hline Missing & 86 (23.9) \\
\hline
\end{tabular}


Table 2: Services Provided by Facilities Willing to Serve Clients with Opioid-related Needs $(N=360)$

\begin{tabular}{|c|c|c|c|}
\hline Facility Characteristics & $\begin{array}{l}\text { Count (\%) / } \\
\text { Mean (SD) }\end{array}$ & Min & Max \\
\hline \multicolumn{4}{|c|}{ SUD Services by level of care (non-mutually exclusive): } \\
\hline Recovery Support Services & $189(52.5)$ & & \\
\hline Recovery management/coaching & $89(24.7)$ & & \\
\hline Peer navigators & $88(24.4)$ & & \\
\hline Recovery housing & $126(35.0)$ & & \\
\hline Recovery community centers/spaces & $2(56.0)$ & & \\
\hline Traditional outpatient & $170(47.5)$ & & \\
\hline Intensive outpatient & $87(24.3)$ & & \\
\hline Day treatment/partial hospitalization & $15(4.2)$ & & \\
\hline Residential & $29(8.1)$ & & \\
\hline Hospital-based inpatient/medical detox & $14(3.9)$ & & \\
\hline Missing & $2(0.0)$ & & \\
\hline Number of SUD services offered & $1.62(0.05)$ & 1 & 6 \\
\hline \multicolumn{4}{|c|}{ Non-SUD services offered (non-mutually exclusive): } \\
\hline Health & $78(21.7)$ & & \\
\hline Mental health & $146(40.7)$ & & \\
\hline Employment/education & $72(20.1)$ & & \\
\hline Legal & $10(2.8)$ & & \\
\hline Case management & $125(34.8)$ & & \\
\hline Interpersonal violence & $34(9.5)$ & & \\
\hline General housing/shelter services & $150(41.8)$ & & \\
\hline Transportation & $38(10.6)$ & & \\
\hline Parenting & $32(8.9)$ & & \\
\hline Family Support & $65(18.1)$ & & \\
\hline Missing & $1(0.0)$ & & \\
\hline Number of non-SUD support services offered & $2.09(0.09)$ & 0 & 8 \\
\hline \multicolumn{4}{|c|}{ Acceptance of Medications for Opioid Use Disorder (OUD) } \\
\hline Zero Acceptance & $88(24.4)$ & & \\
\hline Low Acceptance & $56(15.6)$ & & \\
\hline Moderate Acceptance & $116(32.2)$ & & \\
\hline High Acceptance & $90(25.0)$ & & \\
\hline Missing & $10(2.8)$ & & \\
\hline
\end{tabular}


Table 3: Acceptance of Medications for OUD by Facility Characteristics ( $\mathrm{N}=350)$

\begin{tabular}{|c|c|c|c|c|c|}
\hline \multirow[b]{2}{*}{ Facility Characteristics } & \multicolumn{4}{|c|}{ Facility Acceptance Level of Medications for OUD } & \multirow[b]{2}{*}{$\begin{array}{c}\chi^{2} \\
\text { ( } p \text { value) }\end{array}$} \\
\hline & $\begin{array}{c}\text { Zero } \\
\text { Acceptance } \\
\mathbf{N}=\mathbf{8 8} \\
n(\%)\end{array}$ & $\begin{array}{c}\text { Low } \\
\text { Acceptance } \\
\mathbf{N}=56 \\
n(\%)\end{array}$ & $\begin{array}{c}\text { Moderate } \\
\text { Acceptance } \\
\mathbf{N}=116 \\
n(\%)\end{array}$ & $\begin{array}{c}\text { High } \\
\text { Acceptance } \\
\mathbf{N}=90 \\
n(\%)\end{array}$ & \\
\hline Agency Type & & & & & 33.70 \\
\hline Private, for-profit & $15(11.7)$ & $16(12.5)$ & $45(25.2)$ & $52(40.6)$ & $(<.001)$ \\
\hline Other & 73 (32.9) & $40(18.0)$ & $71(32.0)$ & $28(17.1)$ & \\
\hline Facility focus & & & & & 99.17 \\
\hline SUD only & 67 (39.9) & $26(15.5)$ & $52(31.0)$ & $23(13.7)$ & $(<.001)$ \\
\hline MH/Mixed MH-SUD & $9(6.6)$ & $15(10.9)$ & $51(37.2)$ & $62(45.3)$ & \\
\hline General health & $0(0.0)$ & $0(0.0)$ & $4(44.4)$ & $5(55.6)$ & \\
\hline Other & $12(33.3)$ & $15(41.7)$ & $9(25.0)$ & $0(0.0)$ & \\
\hline Spiritual orientation & & & & & 39.87 \\
\hline Secular & $64(24.4)$ & $29(11.1)$ & $82(31.3)$ & $87(33.2)$ & $(<.001)$ \\
\hline Faith Orientation & $24(27.3)$ & $27(30.7)$ & $34(38.6)$ & $3(3.4)$ & \\
\hline Language services & & & & & 33.32 \\
\hline English only & 80 (32.9) & $40(16.5)$ & $62(25.5)$ & $61(25.1)$ & $(<.001)$ \\
\hline Multiple languages & $8(7.5)$ & $16(15.0)$ & $54(50.5)$ & $29(27.1)$ & \\
\hline Age-specific services & & & & & 22.68 \\
\hline Adult Only & 69 (31.1) & $29(13.1)$ & $59(26.6)$ & 65 (29.3) & $(<.001)$ \\
\hline Other Ages & 19 (14.8) & $27(21.1)$ & $57(44.5)$ & 25 (19.5) & \\
\hline Gender-specific services & & & & & 136.15 \\
\hline Women only & $22(52.4)$ & $9(21.4)$ & $9(21.4)$ & $2(4.8)$ & $(<.001)$ \\
\hline Men only & 45 (67.2) & $13(19.4)$ & $9(13.4)$ & $0(0.0)$ & \\
\hline Both women \& men & $21(8.7)$ & $34(14.1)$ & $98(40.7)$ & $88(36.5)$ & \\
\hline Inpatient/Medical Detox & & & & & 17.94 \\
\hline Not Present & $88(26.2)$ & $56(16.7)$ & $112(33.3)$ & $80(23.8)$ & $(<.001)$ \\
\hline Present & $0(0.0)$ & $0(0.0)$ & $4(28.6)$ & $10(71.4)$ & \\
\hline Day Tx/Residential & & & & & 8.66 \\
\hline Not Present & $81(26.3)$ & $54(17.5)$ & $96(31.2)$ & $77(25.0)$ & $(.034)$ \\
\hline Present & 7 (16.7) & $2(4.8)$ & $20(47.6)$ & $13(31.0)$ & \\
\hline Outpatient/IOP Services & & & & & 54.35 \\
\hline Not Present & $70(39.1)$ & $30(16.8)$ & 32 (17.9) & $47(26.3)$ & $(<.001)$ \\
\hline Present & $18(10.5)$ & $26(15.2)$ & $84(49.1)$ & $43(25.1)$ & \\
\hline Recovery support services & & & & & 106.05 \\
\hline Not Present & $11(6.6)$ & $19(11.4)$ & $57(34.3)$ & $79(47.6)$ & $(<.001)$ \\
\hline Present & $77(41.8)$ & $37(20.1)$ & $59(32.1)$ & $11(6.0)$ & \\
\hline Number SUD services & & & & & 48.71 \\
\hline 1 service only & $72(33.3)$ & $48(22.2)$ & $53(24.5)$ & 43 (19.9) & $(<.001)$ \\
\hline $2+$ services & 16 (11.9) & $8(6.0)$ & $63(47.0)$ & $47(35.1)$ & \\
\hline Number non-SUD services & & & & & 26.15 \\
\hline 0 to 1 services & $69(28.7)$ & $27(11.3)$ & $70(29.2)$ & $74(30.8)$ & $(<.001)$ \\
\hline $2+$ services & 19 (17.3) & $29(26.4)$ & $46(41.8)$ & 16 (14.5) & \\
\hline
\end{tabular}

Note: All cases with missing information were excluded from all analysis $(n=10)$. 
Table 4: Adjusted relative risk ratios (RRRs) and 95\% confidence intervals (Cls) from multinomial logistic regressing the probability of facility acceptance level on facility characteristics $(N=350)$

\begin{tabular}{|c|c|c|c|}
\hline \multirow[b]{2}{*}{ Facility Characteristics } & \multicolumn{3}{|c|}{ Relative Risk Ratios (RRRs) and 95\% Confidence Intervals } \\
\hline & $\begin{array}{l}\text { Zero Acceptance } \\
\qquad N=88\end{array}$ & $\begin{array}{l}\text { Low Acceptance } \\
\qquad N=56\end{array}$ & $\begin{array}{l}\text { High Acceptance } \\
\qquad \mathrm{N}=90\end{array}$ \\
\hline Primary mental health & $0.39[0.16,0.96]^{*}$ & $0.75[0.32,1.76]$ & $3.22[1.57,6.62]^{* *}$ \\
\hline Adult only services & $0.90[0.41,1.99]$ & $0.50[0.23,1.12]$ & $5.12[2.44,10.77]^{* * *}$ \\
\hline Recovery support services & $7.91[3.24,19.28]^{* * *}$ & $2.84[1.18,6.86]^{*}$ & $0.13[0.06,0.29]^{* * *}$ \\
\hline Multiple SUD services & $0.17[0.08,0.38]^{* * *}$ & $0.10[0.04,0.24]^{* * *}$ & $2.86[1.36,6.01]^{* *}$ \\
\hline Multiple non-SUD services & $0.49[0.23,1.03]$ & $1.39[0.67,2.90]$ & $0.49[0.23,1.05]$ \\
\hline State 1 & $2.61[1.31,5.19]^{* *}$ & $0.77[0.36,1.64]$ & $0.86[0.44,1.67]$ \\
\hline Intercept & $0.51[0.17,1.59]$ & $0.83[0.31,2.21]$ & $0.19[0.07,0.55]^{* *}$ \\
\hline \multicolumn{4}{|l|}{ Fit Statistics } \\
\hline Intercept-only log-likelihood & -474.452 & & \\
\hline Final model log-likelihood & -342.487 & & \\
\hline Likelihood Ratio $\chi^{2}(\mathrm{df}=21)$ & $263.929 * * *$ & & \\
\hline
\end{tabular}


Table 5: Rationale Provided by Facility Representative to Justify Acceptance Level $(N=89)$

\begin{tabular}{lcccc}
\hline & Facility Acceptance Level of Medications for Opioid Use Disorder \\
\cline { 2 - 5 } & $\begin{array}{c}\text { Zero } \\
\text { Acceptance }\end{array}$ & $\begin{array}{c}\text { Low } \\
\text { Acceptance }\end{array}$ & $\begin{array}{c}\text { Moderate } \\
\text { Acceptance }\end{array}$ & $\begin{array}{c}\text { High } \\
\text { Acceptance }\end{array}$ \\
Category/Sub-Category & $N=16$ & $N=50$ & $N=25$ & $N=8$ \\
\hline Alcohol/drug free facility & 10 & 5 & 0 & 0 \\
No narcotics allowed & 10 & 0 & 0 & 0 \\
Concern re: community impact & 0 & 5 & 0 & 0 \\
Provider preference & 0 & 5 & 0 & 4 \\
Drug-specific preferences & 0 & 7 & 0 & 0 \\
Injectable Vivitrol only & 0 & 5 & 0 & 0 \\
Injectiabel Vivitrol or & 0 & 1 & 0 & 0 \\
Methadone okay & & & & \\
Short-term medication use only & 0 & 1 & 0 & 0 \\
Prior experiences & 2 & 21 & 0 & 0 \\
Bad experience & 2 & 21 & 5 & 0 \\
Good experience & 0 & 0 & 18 & 4 \\
Level of supports & 4 & 12 & 0 & 0 \\
Insufficient supports/training & 4 & 11 & 12 & 4 \\
Sufficient supports/training & 0 & 0 & 6 & 0 \\
Outside support required & 0 & 1 & 4 & 0 \\
Does not assess for medications & 0 & 0 & & \\
\hline
\end{tabular}

